33 IMPROVING REPORTING OF MEDICATION ERRORS AT AL-WAZARAT PRIMARY HEALTHCARE PHARMACY OF PRINCE SULTAN MILITARY MEDICAL CITY (PSMMC), RIYADH, SAUDI ARABIA

Maather Aljuhani, Khaled Al-Surimi. Pharmacy, Prince Sultan Military Medical City

\subsection{6/bmjoq-2019-PSF.33}

Background It has been found that around $70 \%$ of medication errors (MEs) have been corrected by pharmacists; however, the pharmacists did not report the MEs to the safety reporting system. When we analyzed the existing data, we noticed that the current reporting of MEs by pharmacists was zero, even though the benchmark for the 8-hour shift is estimated to report at least five MEs per day. Thus, we decided to develop an improvement project aiming to increase the number of MEs reported to 64 by November 2018 in Al-Wazarat Primary Healthcare Pharmacy of Prince Sultan Military Medical City (PSMMC).

Methods This quality improvement project was done at AlWazarat (Primary Healthcare Pharmacy) at PSMMC. The Quality Team (QT) has been formulated and started analyzing the reporting data available in the pharmacy department. Data showed there is a huge gap between the number of MEs corrected by pharmacists and their reporting behavior. Then the QT conducted several sessions using quality tools, such as process mapping, brainstorming, and cause-effect techniques, to explore the possible factors causing pharmacists to not report MEs. Several PDSA (plan-dostudy-act) cycles were used to test ideas for change, including redesigning the $\mathrm{ME}$ form to simplify and standardize the reporting process of MEs. The impact of such an intervention had been assessed using the process and outcomes measures. The final results have been analyzed and presented using a run chart.

Results The implementation led to remarkable improvement. In November, 130 MEs had been reported by pharmacists, which exceeded our goal for ME reporting in the Al-Wazarat Primary Healthcare Pharmacy. The number of MEs reported increased from zero in October (before the intervention) to between five and ten MEs reported per day during the month of November after the intervention. Also, the percentage of pharmacists who became active in reporting MEs improved after several PDSA cycles had taken place.

Conclusion Simplification and standardization of the ME form has led to an increase in the reporting of MEs among primary healthcare pharmacists. However, such an intervention might not be sufficient to sustain the pharmacists' new reporting behavior without making such change a part of the pharmacy management safety system. Thus, before spreading these initiatives to another primary healthcare pharmacy, further testing among other pharmacies in a different setting is highly recommended.

\section{ACHIEVING AND SUSTAINING ZERO CLABSI EVENTS IN ONCOLOGY PATIENTS BY IMPLEMENTING TARGETED INTERVENTIONS}

Asim Al Saedi, Wasil Jasthaniah, Muhammad Yaseen, Maher AlAhmadi, Roxanne Taguas, Elma Tana, Omer Abuskout, Osama Younis, Mareun Daniel, Javaid Bhutta, Majid AlShamrani. Infection Prevention and Control King Abdulaziz Medical City
Background Central line-associated bloodstream infections (CLABSIs) are one of the potentially life-threatening complications that occur in patients with cancer. Central lines are usually required in these patients for prolonged durations. The purpose of this study was to identify and implement multiple interventions for the prevention of CLABSIs in patients with cancer.

Methods A multidisciplinary taskforce was created to identify and implement evidence-based interventions to achieve a target of zero CLABSI in both adult and pediatric oncology patients. Monitoring of CLABSI and central line bundle also continued as it was being done before creation of the taskforce. These interventions included mandatory educational sessions, use of a dedicated trolley for central line use only, patient and family education on care of central lines, chlorhexidine bath before insertion of central lines, review of staff competency on handling central lines, and improvement in the completion of central line bundle. These interventions were introduced at three levels of line management: pre-insertion, during insertion, and post-insertion of central lines. Data were collected on a daily basis with analysis and reporting on a quarterly basis.

Results In adult patients, the quarterly rate of CLABSI in the four quarters of 2017 was 1.9, 1.9, 1.3, and 2.1 per 1000 central line days, respectively. The overall annual rate in the year 2017 was 1.8 per 1000 central line days with a total of seven CLABSI events and 3875 central line days. For pediatric patients, the quarterly rate of CLABSI in the four quarters of 2017 was $1.3,1.2,1.2$, and 1.1 per 1000 central line days, respectively. The overall annual rate in the year 2017 was 1.2 per 1000 central line days with a total of eight CLABSI events and 6638 central line days. As a result of the interventions, no CLABSI events were observed in either adult or pediatric patients in the first two quarters of 2018.

Conclusion A collaborative effort by the dedicated multidisciplinary team resulted in achieving zero CLABSI. The targeted interventions resulted in achieving the ultimate goal of zero CLABSI and sustaining it for 6 months in high-risk oncology patients.

\section{RISK FACTORS FOR ACQUISITION OF MULTIDRUG- RESISTANT GRAM-NEGATIVE BACTERIA IN A TERTIARY CARE HOSPITAL IN SAUDI ARABIA: A CASE-CONTROL STUDY}

AbdulHakeem AlThaqafi, Muhammad Yaseen, Fayssal Farahat, Abdulfattah Mowallad, Sabiha Essack. Infectious Diseases, Internal Medicine, King Abdulaziz Medical City, Jeddah

\subsection{6/bmjoq-2019-PSF.35}

Background The increase in incidence of multidrug-resistant (MDR) organisms, especially gram-negative bacteria, in healthcare facilities is a serious cause of concern worldwide. This study was done at King Abdulaziz Medical City, Jeddah, a tertiary care hospital. The World Health Organization has published a priority pathogens list of antibiotic-resistant bacteria. The priority pathogens have been categorized into three major priorities (i.e., critical, high, and medium). The critical priority pathogens include common gram-negative bacteria (GNB) such as carbapenem-resistant Acinetobacter baumannii, carbapenemresistant Pseudomonas aeruginosa, and carbapenem-resistant and third-generation cephalosporin-resistant Enterobacteriaceae such as Klebsiella pneumoniae and Escherichia coli. The 
current study identified risk factors for the acquisition of these MDR gram-negative critical priority pathogens in King Abdulaziz Medical City, Jeddah, to inform strategies for their containment.

Methods A case-control study was carried out from January to April 2015, in which 100 patients with healthcare-associated infections (infections arising 48 hours after admission) caused by MDR GNB were compared with two control groups, i.e., 100 patients with healthcare-associated infections caused by non-MDR GNB (not meeting the criteria of MDR) and 100 patients without infection caused by GNB. MDR bacteria were defined as bacteria that were non-susceptible to at least one antibiotic in three or more classes of antibiotics. Data were analyzed using descriptive statistics (frequency and percentage of categorical variables). Multivariate regression analysis was undertaken to identify significant predictors of MDR GNB. Odds ratios (ORs) with 95\% CIs were calculated and the level of significance was determined as $\mathrm{p}<0.05$.

Results A total of 388 organisms were isolated during the study period from 332 patients. 56 (14\%) patients were infected with more than one organism. Antibiotic therapy (OR 5.50, 95\% CI 2.19-13.84; OR 3.98, 95\% CI 1.68-9.44), stay in intensive care unit (OR 11.11, 95\% CI 4.58-26.93; OR 8.60, 95\% CI 3.28-22.57), and having indwelling medical devices (OR 3.02, 95\% CI 1.45-6.33; OR 2.43, 95\% CI 1.11-5.33) were the significant risk factors in patients infected with MDR GNB compared with each of the other two control groups, respectively.

Conclusion The risk factors identified in our study provide guidance for healthcare workers for the prevention and containment of MDR GNB with special emphasis on effective implementation of an antimicrobial stewardship program and enhancing infection control practices in intensive care units.

\section{THE EFFECTIVENESS OF STERILE TECHNIQUE IN REDUCING FALSE-POSITIVE BLOOD CULTURE RESULTS IN QATIF CENTRAL HOSPITAL EMERGENCY DEPARTMENT}

Mohammed AL-Bazroun, Auday ALKhunaizi, Arif Al-Hamad, Ali Ismail, Zahra Talib, jaffar AL- Twoailb, Maha al Ibrahim, M Alquraini. Emergency Department/Laboratory Department Qatif Central Hospital

\subsection{6/bmjoq-2019-PSF.36}

Background Blood culture contamination is a common and preventable problem in the emergency department (ED). In previous studies, changing the process of ED blood culture collection into a more sterilized procedure $r$ esulted in a substantial reduction in the rate of blood contamination. The present study assessed the degree of blood contamination and evaluated the effect of using a sterile technique with monitoring and feedback on contamination rate over a 1-year period.

Methods We documented the rate of blood contamination among blood samples sent from the ED in the period from January 2016 until March 2016. A workshop for all ED nurses was held in March 2016 by clinical nurse instructors and this was followed by daily bedside teaching sessions for the whole study period. Nurses were instructed and audited on proper sterile blood withdrawal techniques. During the intervention period, we measured the rate of blood contamination for the period from April 2016 until September 2016. Results Our average contamination rate dropped from the baseline of $12.6 \%$ (58 out of a total of 736 samples) to an average contamination rate of approximately 5.6\% (122 out of a total of 1549 samples), with an odds ratio of 0.411 (95\% CI 0.303-0.559; p<0.001).

Conclusion Changing the method of blood culture collection from the commonly used aseptic technique to a sterile process resulted in significant reductions in blood culture contamination in a busy community hospital ER. Monitoring the implementation process was important to identify and overcome operational challenges. In addition, this study could be a good initiative to start a multicentric quality improvement project to reduce blood contamination in the neighboring community and public hospitals.

\section{MEDICATION EXCHANGE AND SHARING NETWORK PROGRAM (MESNP) INITIATIVE TO COPE WITH DRUG SHORTAGES IN THE KINGDOM OF SAUDI ARABIA (KSA)}

Aeshah AlAzmi, Rph Faris AlRashidi. Pharmaceutical Care Services Department, King Abdulaziz Medical City Jeddah

\subsection{6/bmjoq-2019-PSF.37}

Background Drug shortages are a major public health concern and remain a persistent problem worldwide. Saudi Arabia is one of the richest and fastest growing countries in the Middle East. Despite that, Saudi Arabian drug markets are not immune to drug shortages. Although exact figures about drug shortages in Middle Eastern countries in general and in Saudi Arabia, in particular, are lacking, there is an emerging yet still limited number of reports about the drug shortage. It is, however, a fact that the drug shortages are affecting the Middle East in general and the Kingdom of Saudi Arabia (KSA) in particular. At the time we conducted this project, the Saudi Food and Drug Administration (SFDA) had not yet fully activated and implemented its role in tracking drug shortages and the role of other regulatory bodies were either outdated or unknown. Healthcare is one of the main focus areas of Saudi Vision 2030, which represents a comprehensive plan for the entire economic structure of Saudi Arabia. In order to ensure the Saudi Vision 2030 becomes reality, we should focus on more efficient use of our current resources. Based on that, we identified an innovative solution at the national level to collaborate and cope with the current situation by developing a centralized Medication Exchange and Sharing Network Program (MESNP).

Methods A quality improvement process map method was used for this project. Baseline evaluation included a review of possible reasons and strategies to manage medication shortages, recognize potential associated safety issues, and we developed MESNP as a national novel project to cope with medication shortages using a telegram as the preferred social media platform for group creation and communication.

Results A total of 500 reports were received. The majority of reports $(70 \%)$ were raised by the Ministry of Health $(\mathrm{MOH})$. A number of reports constituted requests for drug supplies due to shortages $(n=315)$ and reports indicating the availability of overstock items for redistribution $(n=185)$. Almost 98\% of overstocking drug reports were redistributed, which covered $75 \%$ of drug shortage requests.

Conclusion We believe that this is the first national novel project aiming to address drug shortages. The optimistic findings of this project were the proactive identification of data and development of a framework to collect data about 